\title{
lincRNA-RP11400K9.4 Regulates Cell Survival and Migration of Breast Cancer Cells
}

\author{
MIGUEL A. FERNÁNDEZ-ROJAS ${ }^{1}$, JORGE MELENDEZ-ZAJGLA ${ }^{2}$ and VILMA MALDONADO LAGUNAS ${ }^{1}$ \\ ${ }^{1}$ Epigenetics Laboratory, Instituto Nacional de Medicina Genómica, México City, México; \\ ${ }^{2}$ Functional Genomics Laboratory, Instituto Nacional de Medicina Genómica, México City, México
}

\begin{abstract}
Background/Aim: Several works in the past decades pointed out the key role of long intergenic non-coding RNA (lincRNA) in breast cancer development. Here in we report for first time the importance of deregulation of lincRNA RP11$400 K 9.4$ in breast cancer cells which played a role in cell survival and migration. Materials and Methods: After RP11400K9.4 silencing by short hairpin RNAs or overexpression by GeneBlocks, real-time quantitative polymerase chain reaction $(R T-P C R)$, microarray, migration, proliferation and viability assay were performed. Results: RP11-400K9.4 expression was mainly in the cytoplasmic fraction in $2 D$ culture. Overexpression of RP11-400K9.4 led to a reduction of migration by MCF-7 and $M D A-M B-368$ cells and an increase in cellular survival after $U V-C$ radiation. Bioinformatic analyses highlighted irradiationinduced DNA damage, DNA repair and cell-cycle pathways as the mainly affected by RP11-400K9.4. Furthermore RT-PCR assay demonstrated the overexpression of baculoviral IAP repeat containing 3 (BIRC3) a known oncogene that promotes radiotherapy resistance through the nuclear factor kappa $B$ $\left(N F_{k} B\right)$ pathway. Conclusion: RP11-400K9.4 participates in the modulation of migration and survival processes probably via the BIRC3/NFKB pathway.
\end{abstract}

Breast cancer is a worldwide health problem, representing the most common life-threatening malignant neoplasm in women and the second cause of death associated with cancer (1). In the case of Mexico, breast cancer is also the most common type in women, and its mortality has been increasing in recent years; this tendency is expected to continue in the coming years (2).

This article is freely accessible online.

Correspondence to: Vilma Maldonado, Ph.D., Epigenetics Laboratory, National Institute of Genomic Medicine, México City, México. Tel: +52 5553501900, e-mail: vmaldonado@inmegen.gob.mx

Key Words: Breast cancer, lincRNA-RP11400K9.4, cell survival, migration, BIRC3.
The cancer cell phenotype is a dynamic state in which cells modify distinctive and complementary capabilities such as proliferation, evasion of cell growth signals, sensitivity to death via different external and internal stimuli, migration and invasion (3).

Resistance to death constitutes a genuine hallmark of cancer and is a major obstacle in the treatment of cancer. The sensitivity of a cell to death from external and internal stimuli depends on specific molecules and transduction pathways regulated at the translational and post-translational levels. The inhibitors of apoptosis (IAP) are a family of functionally and structurally related proteins that serve as endogenous inhibitors of programmed cell death and play a very important role in cancer cells obtaining resistance to death (4). A common feature of IAPs is the presence of a baculovirus IAP repeat (BIR), a 70 amino acid domain, in one to three copies which function mainly by mediating protein-protein interactions (5). This family comprises BIRC1 (NAIP), BIRC2 (cIAP1), BIRC3 (cIAP2), BIRC4 (xIAP), BIRC5 (survivin) and BIRC6.

BIRC3 can directly bind to caspases 3, 7, and 9 to inhibit their activation (6). This protein has also been implicated in the activation of several signal transduction pathways associated with malignancy (7). BIRC3 was found to be upregulated in breast cancer cells stimulated with interleukin-1 $\beta$, and this change is associated with resistance to doxorubicin, whereas silencing of BIRC3 reduced the viability of cells after exposure to the drug (8). BIRC3 plays also an important role in regulating the nuclear factor- $\mathrm{kB}(\mathrm{NF}-\mathrm{kB})$ signaling pathway, which is involved in the development of cancer (9). Recently, it was shown that this protein can be associated with noncoding RNAs: binding of miR-124 to BIRC3 inhibited the proliferation and migration of in human hepatocellular carcinoma (10). Interestingly, in 2019, Jiang et al. reported that the long non-coding RNA IncRNA-HCP5 promoted triple-negative breast cancer progression, regulating BIRC 3 by binding to miR-219a-5p (11).

After the discovery of lncRNA in eukaryotic cells in 1986, work has shown their importance as genetic and epigenetic regulators in tumor cells and therefore in the acquisition of 
hallmarks or emergent neoplastic characteristics $(12,13)$. Various studies have shown a role of lncRNA in the regulation of migration and death resistance, such as CCND1-upstream intergenic DNA repair 1 (CUPPID1) and 2, nuclear paraspeckle assembly transcript 1 (NEAT1), and DNA damage-sensitive RNA1 (DDSR1) (14-17). Recently, long intergenic non-coding RNA (lincRNA) RP11400k9.4 was pointed out as a possible marker for triple-negative breast cancer (18-20). In addition, we found that this lincRNA is associated with deregulation of inhibitor of nuclear factor kappa B kinase subunit epsilon (IKKe) kinase and the stem cell phenotype in breast cancer (21). In this study, we aimed to elucidate the role of this lincRNA in breast cancer cells.

\section{Materials and Methods}

Cell culture. Cells lines MCF-7, T47D, ZR751, MDA-MB-361, MDA-MB-453, MDA-MB-468, MDA-MB-231, SKBR3, HS578T and BT-20 were obtained from the American Type Culture Collection (ATCC, Manassas, VA, USA) and maintained at $37^{\circ} \mathrm{C}$ with $5 \%$ of $\mathrm{CO}_{2}$ in Dulbecco's modified Eagle's medium (DMEM), Eagle's minimum essential medium (EMEM) or Roswell Park Memorial Institute (RPMI) medium with fetal bovine serum (FBS) at $5-15 \%$, according to recommendations from the ATCC.

Cellular transfection. To generate cell lines that stably overexpress lincRNA RP11-400k9.4, MCF-7 and MDA-MB-468 cells were transfected with $7.5 \mathrm{ml}$ of lipofectamine and $3 \mu \mathrm{g}$ of the pcDNA3.1 vector containing the complete cDNA of the lincRNA GB400 (GeneBlock for RP11-400k9.4) (gBlocks ${ }^{\circledR}$ Gene fragments, IDT, Newark, NJ, USA), As transfection control, we used the same vector with a Luciferase insert. These cells were selected with 600 and $800 \mu \mathrm{g} / \mathrm{ml}$ of 418 Geneticin (Thermo Fisher Scientific, CDMX, México) respectively. For the loss-of-function experiments, two short hairpin RNAs (shRNAs) were designated with the software RNAi Tool (Clontech, Takara, CA, USA). The oligonucleotides with the sequence of shRNA or a Luciferase sequence were inserted in the pSiren RQ vector (Clontech) and $3 \mu \mathrm{g}$ of vector and $7.5 \mathrm{ml}$ lipofectamine were used for the transfection. Cells were selected with $400 \mathrm{ng} / \mathrm{ml}$ puromycin (GIBCO, Gaithersburg, MD, USA).

Quantitative real-time polymerase chain reaction (RT-PCR). Trizol reagent was employed for total RNA isolation of transfected cells. 1 $\mu \mathrm{g}$ of RNA previously treated with DNAse was used for retrotranscription with High Capacity cDNA Reversion Transcription kit (Thermo Fisher Scientific, Franklin Lakes, MA, USA). cDNA (50 ng) was mixed with $7 \mu \mathrm{l}$ of Sybr Select Master Mix (Thermo Fisher Scientific) and $0.24 \mathrm{mM}$ of each primer Supplementary Table I (https://www.dropbox.com/sh/z82pwriywvwjw7f/AADPvyeuQTLKPi xFjuyvSG0ba/Suplementary\%20table\%20I.docx?dl=0), and the assays were performed using a QuantStudio 7 Flex Real Time PCR system. Data were analyzed with QuantStudio Real-Time PCR software (Thermo Fisher Scientific, Franklin Lakes, MA, USA) according to the $2^{-\Delta \Delta C T}$ method (22).

Cellular fractionation. Nuclear and cytoplasmic fractions of MCF7 cells were obtained with PARIS kit (Thermo Fisher Scientific) according to the manufacturer instructions. Total RNA and protein from both fractions were extracted. The purity of the fractions was corroborated with RNA electrophoresis. The protein fraction was separated with sodium dodecyl sulfate-polyacrylamide gel and transferred to a polyvinylidene fluoride membrane. Finally, the membrane was incubated with antibodies toward tubulin and $\mathrm{A} / \mathrm{C}$ lamin (ab15246, ab224816; Abcam, Cambridge, UK) for cytoplasmic and nuclear fractions, respectively.

Extraction of total RNA. MCF-7 and MDA-MB-468 cells were lysed with $1 \mathrm{ml}$ Trizol reagent, $200 \mu \mathrm{l}$ chloroform was added and was then vortexed, after centrifuging at $8000 \times g$ for $12 \mathrm{~min}$. The aqueous phase was transferred to a fresh tube and was mixed with $0.5 \mathrm{ml}$ isopropyl alcohol and then the bottom was recuperated by centrifugation at $10,000 \times g$ for $10 \mathrm{~min}$ and dissolved with $40 \mu \mathrm{l}$ of water.

RNA microarray and pathway analysis. The quality of total RNA was evaluated using an Agilent RNA Bioanalyzer and only RNA of samples with RNA integrity number (RIN) of 9 or higher were used. Three independent samples of MCFF7 cells overexpressing lincRNA RP11-400K9.4 and three of cells transfected with control luciferase were treated with DNAse and hybridized to Affymetrix Gen 2.0 Human Gene slides (Thermo Fisher Scientific) on INMEGEN Microarray Department. Analysis was performed with the Transcriptome Analysis console (Affymetrix/Thermo Fisher Scientific, Waltham, MA, USA). Only genes with a fold change threshold of \pm 3 or higher and $p \leq 0.05$ were considered for analysis with Key Pathway Advisor software (KPA; Clarivate Analytics, Philadelphia, PA, USA),

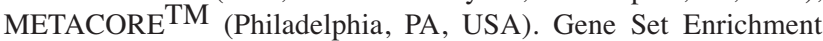
Analysis (GSEA; http://www.broadinstitute.org/gsea) was performed by applying Hallmark and KEGG gene sets downloaded from MSigDB database. We used default settings and considered a false discovery rate of $<0.25$ as statistically significant (23). Likewise, REVIGO (24) (http://revigo.irb.hr/) and Enrichr(25) (https://amp. pharm.mssm.edu/Enrichr/) analyses were performed with default settings (medium similarity $=0.7$ and calculated $p$-value for Gene Ontology predicted pathways). This allowed us to obtain a scatterplot of affected biological processes such as mammary gland development, apoptosis, irradiation-inducedDNA damage, by REVIGO and a table of main deregulated signaling pathways such as ATR by combining the BioPlanet 2019, NCI-Nature 2016 and WikiPathways 2019 Human databases using Enrichr software

Multicellular spheroid formation. A total of $2 \times 10^{6}$ MCF-7 cells were cultivated in non-adherent plates with Leibovitz's L-15 media and incubated with $60 \mathrm{rpm}$ orbital agitation at $37^{\circ} \mathrm{C}$. Spheroids were counted and measured at 21 days and finally disaggregated with trypsin. Total RNA was isolated as described above.

Migration assay. MCF-7 and MDA-MB-368 cells were seeded at $3 \times 10^{5}$ on inserts of transwell plates (Corning, NY, USA) without fetal bovine serum (FBS) overnight. The medium was replaced by fresh medium after 12 hours and medium with $10 \%$ FBS was placed on the bottom wells. After 24 hours, the cells were fixed with $4 \%$ paraformaldehyde and stained with Gentian violet at $10 \%$. Finally, the insert membranes were allowed to dry and the cells that had migrated across the membrane were counted with a stereoscopic microscope and analyzed using ImageJ software (https://imagej.nih.gov/ij/index.html). Proliferation assay. MCF-7 cells were seeded $3 \times 10^{3}$ on each well of a 96-well plate (Corning). DMEM with 5\% FBS was added and the cells maintained at standard temperature and $\mathrm{CO}_{2}$ conditions. 
Table I. Top 10 deregulated RNAs after forced overexpression of lincRNA-RP11400K9.4 in MCF-7 cells.

\begin{tabular}{|c|c|c|}
\hline ID & Gene & Fold change \\
\hline IFI44L & Interferon induced protein $44-$ like & 89 \\
\hline IFIT1 & Interferon induced protein with tetratricopeptide repeats 1 & 71 \\
\hline OAS2 & 2'-5'-Oligoadenylate synthetase 2 & 62 \\
\hline IFIT3 & Interferon-induced protein with tetratricopeptide repeats 3 & 48 \\
\hline IFI44 & Interferon induced protein 44 & 47 \\
\hline HLA-DRA & Major histocompatibility complex, class II, DR alpha & 43 \\
\hline $\mathrm{CP}$ & Ceruloplasmin & 33 \\
\hline PI3 & Peptidase inhibitor 3 & 30 \\
\hline DDX60 & DExD/H-box helicase 60 & 27 \\
\hline CASP14 & Caspase 14 & 26 \\
\hline HMCN1 & Hemicentin 1 & -8 \\
\hline HIST1H1B & H1.5 Linker histone, cluster member & -6 \\
\hline LINC00052 & Long intergenic non-protein coding RNA 52 & -6 \\
\hline DKK1 & Dickkopf WNT signaling pathway inhibitor 1 & -6 \\
\hline HEY2 & Hes-related family BHLH transcription factor with YRPW motif 2 & -5 \\
\hline HIST1H2BM & H2B Clustered histone 14 & -5 \\
\hline PEG10 & Paternally expressed 10 & -5 \\
\hline MCM10 & Minichromosome maintenance 10 replication initiation factor & -5 \\
\hline FAM111B & Family with sequence similarity 111 member B & -5 \\
\hline SCIN & Scinderin & -4 \\
\hline
\end{tabular}

The cells were then exposed to 3-(4,5-dimethylthiazol-2-yl)-2,5diphenyltetrazolium bromide (MTT) reagent (Promega, Madison, WI, USA) for 30 minutes in the dark and the dye solubilized for 1 hour. The dye concentration was measured at $0,24,48,72,96,120$ and 144 hours using a Beckman DTX 880 spectrophotometer (Beckman, Indianapolis, IN, USA) at a wavelength of $595 \mathrm{~nm}$.

Viability assay. MCF-7 cells transfected with Luc, shRNA1, shRNA2 and GB400 were irradiated with UV light at $254 \mathrm{~nm}$ for 3 minutes before seeding at $1.5 \times 10^{4} /$ well in three wells of a 96 -well plate (Corning). After $24 \mathrm{~h}$, the cells were stained with MTT (Promega) reagent and absorbance at $595 \mathrm{~nm}$ measured.

Statistical analysis. Statistical analysis was performed with Graph Pad Prism 6 software (San Diego, CA, USA). We considered a minimum $p$-value of 0.05 and used one- and two-tailed ANOVA test and Student $t$-test to determine significant differences between groups.

\section{Results}

LincRNA-RP11400k9.4 general features. lincRNA-RP11400k9.4 (ENSG00000237807(RP11-400K9.4), ENST00 000426023.1 (RP11-400K9.4-001), lncOPRK1-3:12) is a long non-coding RNA of 2387 base pairs, intergenic, with two exons (Exon1: 154435889-54436491, Exon 2: 254427731-54429514), located on chromosome 8 , at 54,427,731-54,436,491 (Supplementary Figure 1A; https://www.dropbox.com/sh/z82pwriywvwjw7f/AA BVYIdJa_Psqbj-SL6z AHXFa/Supplemmentary\%20figure \% 201.pdf?dl=0). It has been shown that lincRNA-RP11400k9.4 is expressed in four normal tissues/cell lines: Heart, testes, ovary and prostate; and in five types of cancer: Invasive breast carcinoma, lung squamous cell carcinoma and low grade glioma
(http://annolnc.cbi.pku.edu.cn/status.jsp?id=5D3C6210200201084703) (Supplementary Figure $1 \mathrm{~B}$ and C; https://www.dropbox.com/sh/z82pwriywvwjw7f/AABVYIdJa_P sqbj-SL6zAHXFa/Supplemmentary\%20figure\%201.pdf?dl=0).

To determine the subcellular distribution of lincRNARP11400k9.4, cellular cytoplasmatic and nuclear fractions of MCF-7 cells were obtained and its levels were determined by RT-PCR in each fraction. As shown in Figure 1A, lincRNARP11400k9.4 was found at highest levels in the cytoplasmatic fraction. This result is coincident with the data on the lncATLAS database (https://lncatlas.crg.eu/) (Figure 1B).

To determine if lincRNA-RP11400k9.4 was characteristic of one of the breast cancer subtypes, expression analysis was performed on 10 cell lines. We found that the expression was not associated with the hormonal receptor status, subtype, claudin level or doubling time (Supplementary Table II; https://www.dropbox.com/sh/z82pwriywvwjw7f/AAAxgWg 8nE155B8eNo4GKWR9a/Supplementary\%20Tablas\%20II.d ocx?dl=0). Interestingly, we found an inverse correlation between invasive capacity and level of lincRNARP11400k9.4. The lowest levels of expression were found in the MDA-MB-361 and HS578T and MDA231 cell lines, which have a high invasive capacity, whereas the highest levels were found in the MDA-MB-453, T47D and MCF-7 cell lines, which have lower invasive capacity (Figure 1C).

Comprehensive transcriptional analysis of MCF-7 overexpressing lincRNA-RP11400k9.4. In a previous study we observed a decrease in the level of several lncRNAs, including lincRNA-RP11400k9.4, in MCF-7 cells which had acquired a 
A

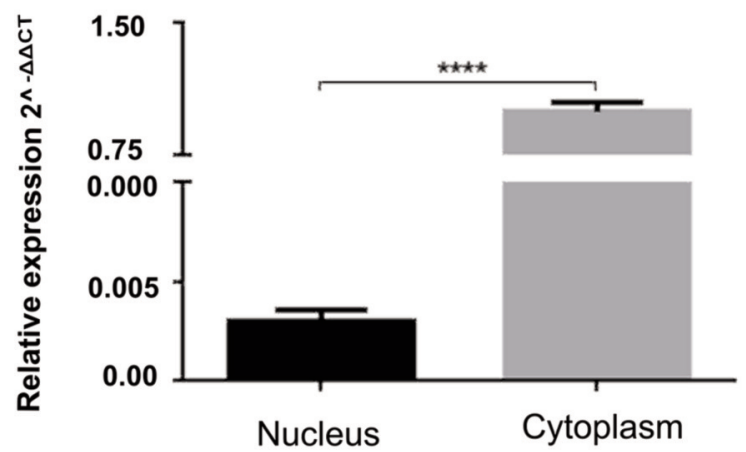

B

RP11-400K9.4 : MALAT1

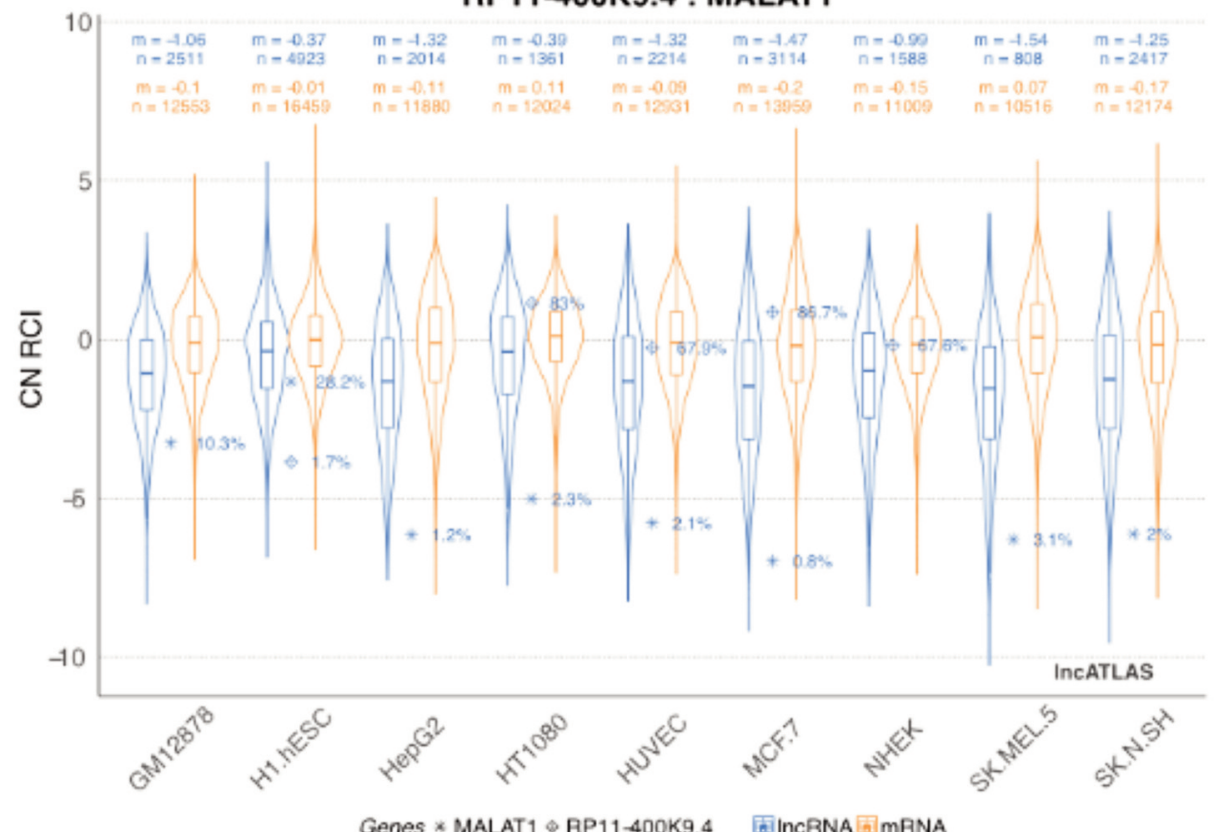

C

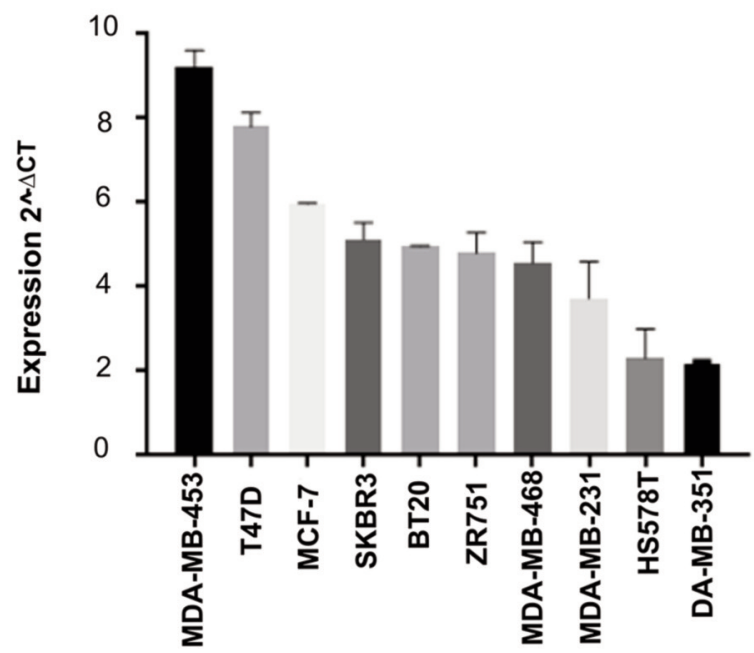

Figure 1. Subcellular localization of long intergenic non-coding RP11-0400k9.4 (lincRNARP11-400k9.4). A: Subcellular distribution of lincRNA RP11-400k9.4 in MCF-7 cells. B: Data of the lncATLAS database of subcellular relative expression of lincRNA RP11-400k9.4 and metastasisassociated lung adenocarcinoma transcript 1 (MALAT1) as positive control of the analysis in MCF-7 and several other cell lines. C: Expression of lincRNA in a panel of 10 cell lines. All data are shown as the mean with \pm SEM from three independent experiments. Student's $t$-test was performed to evaluate differences. $* * * *$ Significantly different at $p=0.0001$. 
A

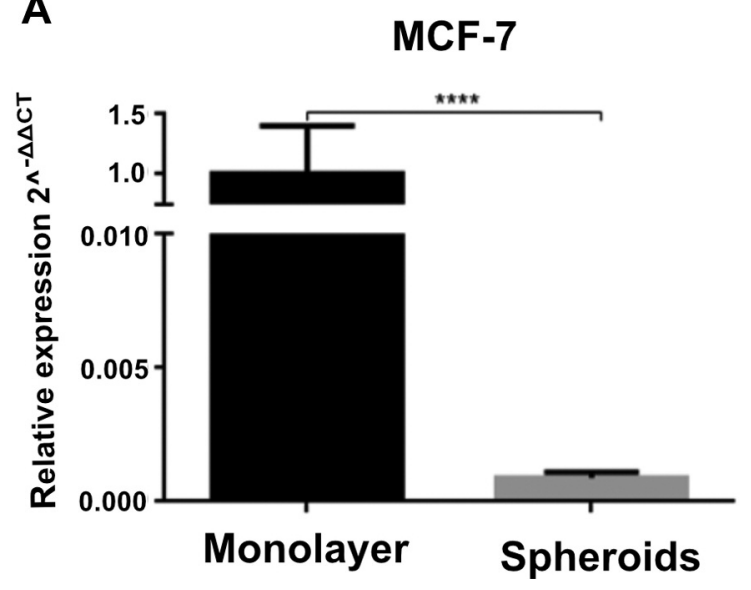

C

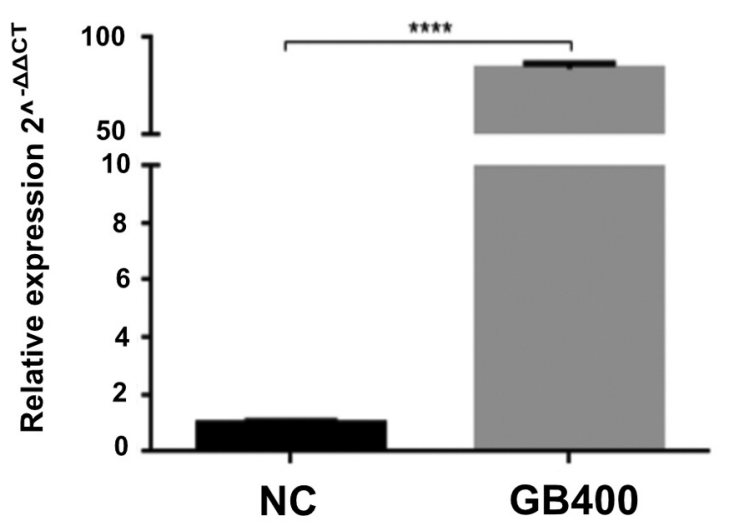

B

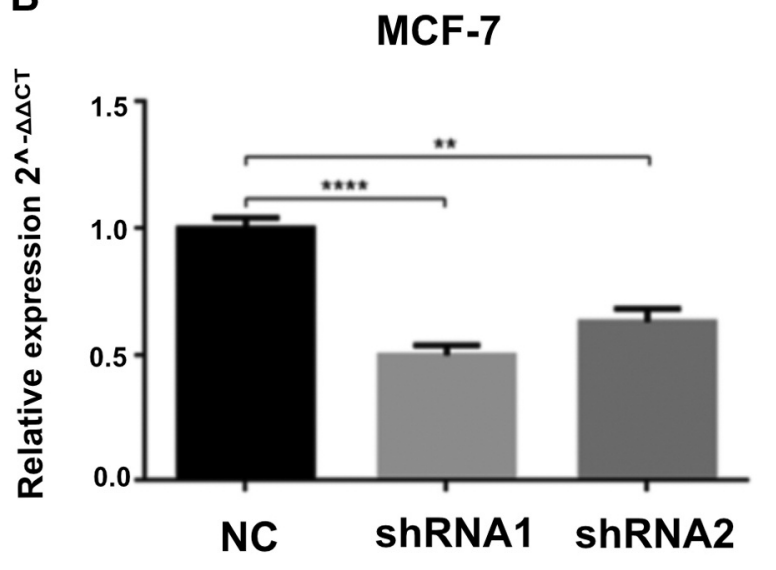

D

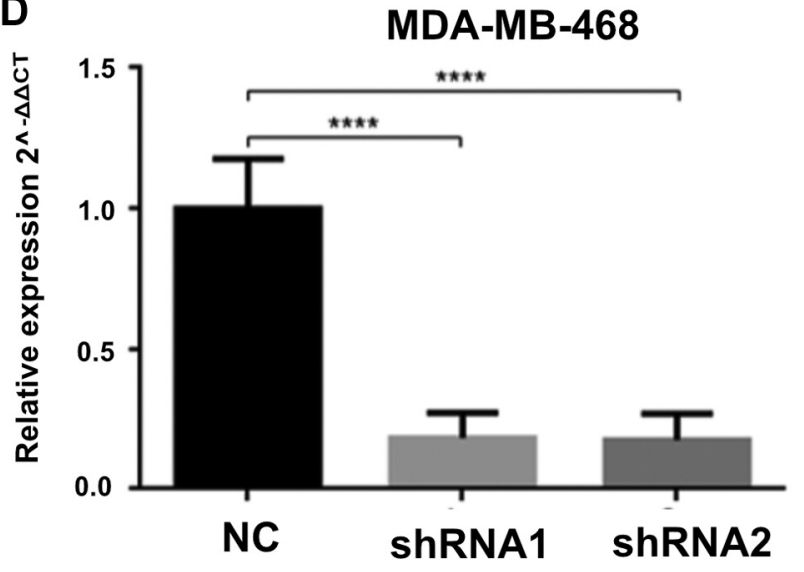

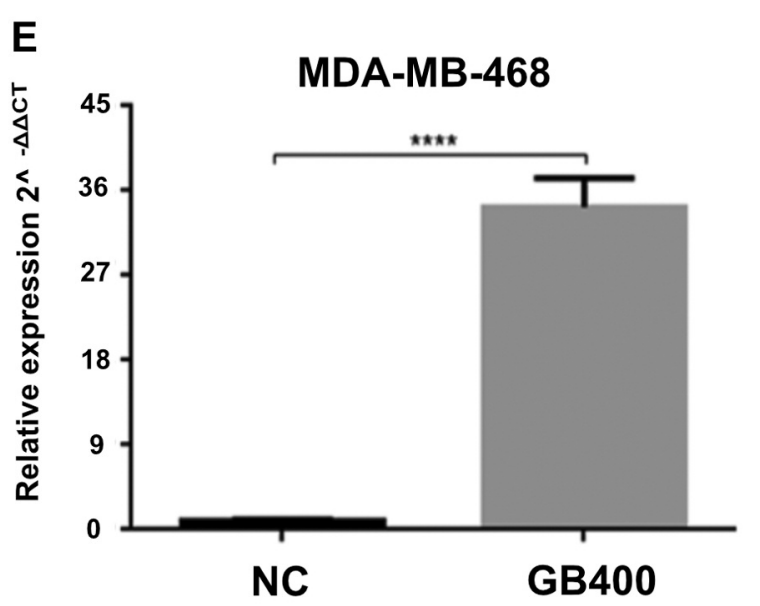

Figure 2. Long intergenic non-coding RP11-0400k9.4 (lincRNARP11-400k9.4) expression in MCF-7 cells. A: Relative expression of lincRNARP11$400 \mathrm{k} 9.4$ in MCF-7 cells growing in $3 D$ (spheroids) in comparison to monolayer cultures. B: Underexpression of lincRNARP11-400k9.4 using two short hairpin RNA (shRNAs) in MCF-7 cells. C: Overexpression of incRNARP11-400k9 unsing GeneBlock for RP11-400k9.4 (GB400) in MCF-7 cells. D: Underexpression of lincRNARP11-400k9.4 using two shRNAs in MDA-MB-468 cells. E: Overexpression of incRNARP11-400k9 using GeneBlock for RP11-400k9.4 (GB400) in MDA-MB-468 cells. All data are show as the mean with \pm SEM from three independent experiments. Student's t-test was performed to evaluate differences. Significantly different at: $* * p=0.01$ and $* * * * p=0.0001$. 
A

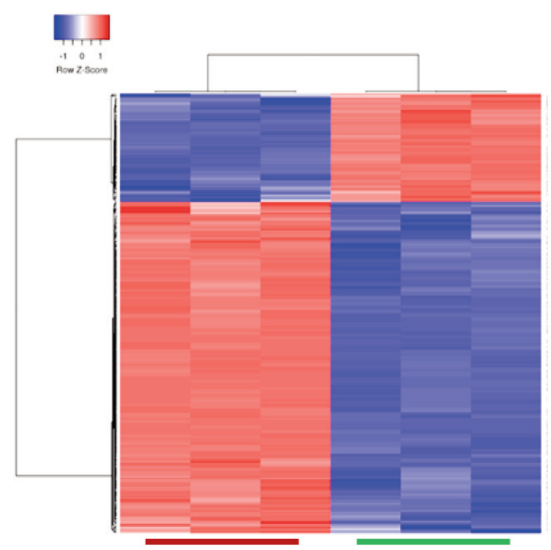

C

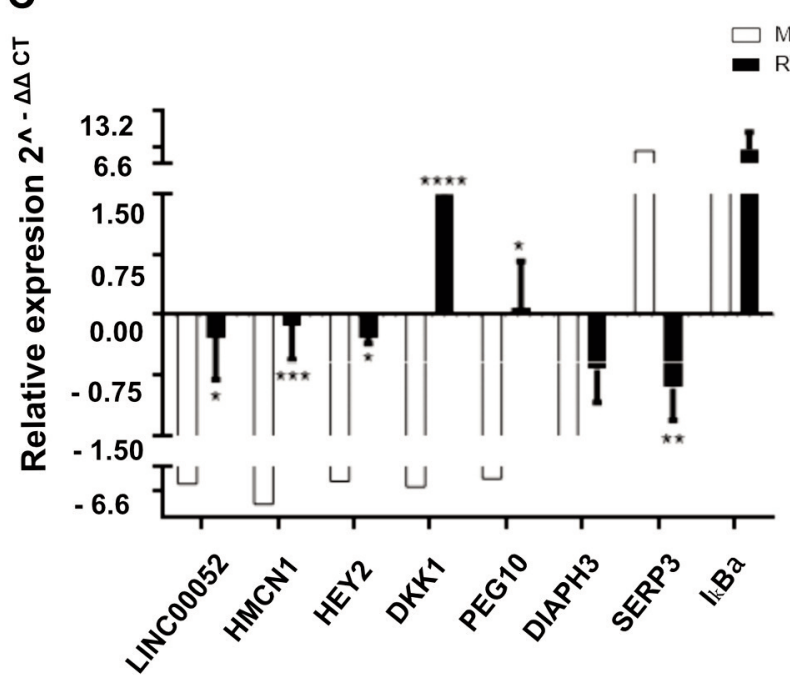

B

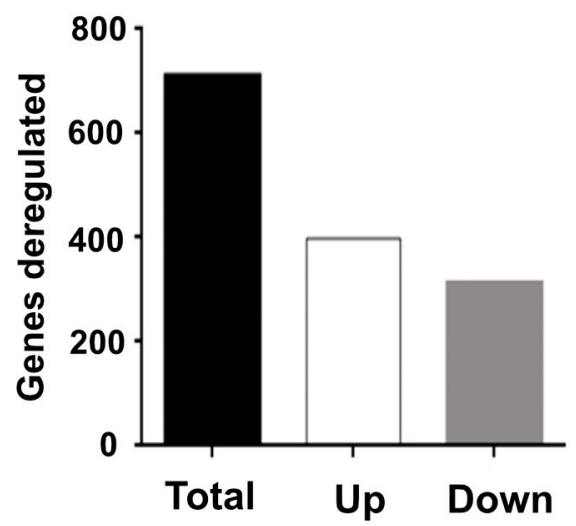

Figure 3. Impact of long intergenic non-coding RP11-0400k9.4 (lincRNARP11-400k9.4) overexpression in MCF-7 cells. A: Heatmap of transcripts altered by lincRNARP11-400k9.4 overexpression. B: mRNAs deregulated in MCF-7 cells with lincRP11-900k.9.4 overexpression. C: Microarray expression data validation of 15 genes by real-time polymerase chain reaction. LINC00052: Long intergenic non-protein coding RNA 52; HMCN1: hemicentin 1; HEY2: Hes-related family BHLH transcription factor with YRPW motif 2; DKK1: Dickkopf WNT signaling pathway inhibitor 1; PEG10: paternally expressed 10; DIAPH3: diaphanous-related formin 3; SERP3: Serpine 3; IkBa: NFKB Inhibitor alpha; SP100: speckled 100 kDa; PI3: peptidase inhibitor 3; IFIT3: interferon-induced protein with tetratricopeptide repeats 3; DDX60: DExD/H-box helicase 60; IFIT1: interferon-induced protein with tetratricopeptide repeats 1; IFIT44L: interferon-induced protein 44-like; BIRC3: baculoviral IAP repeat containing 3. Data shown are the mean \pm SEM from three independent experiments. Two-way ANOVA was performed to evaluate the significance of microarray $v$ s. real time polymerase chain reaction data: Significantly different at: $* p=0.05, * * p=0.01, * * * p=0.001$, and $* * * p=0.0001$.

stem-like phenotype after IKKe was overexpressed (21). It is well known that three-dimensional (3D) $\mathrm{MCF}-7$ culture (spheroids) mimic the main features of human solid tumors including migration, invasion, proliferation, stemness features, and an increase of IKKe level (21). We evaluated the expression of lincRNA-RP11400k9.4 in 3D cultures. As shown in Figure 2A, we found a lower expression of lincRNA-RP11400k9.4 in spheroids when compared to cells cultured in monolayers.

To deepen the understanding of the role of lincRNARP11400k9.4 in breast cancer we performed loss- and gain- of-function experiments by over- and underexpressing lincRNA-RP11400k9.4 in MCF-7 and MDA-MB-468 cells by transfecting two shRNAs (Figure $2 \mathrm{~B}$ and D) or a plasmid with the lincRNA-RP11400k9.4 gene (Figure 2C and E); in both cases we used transfected cells with a control vector to compare the effects.

Firstly, we performed whole-genome expression analysis using MCF-7 cells with overexpression of lincRNARP11400k9.4 versus luciferase expression. The validation of data obtained with the microarray analysis was performed using 
A

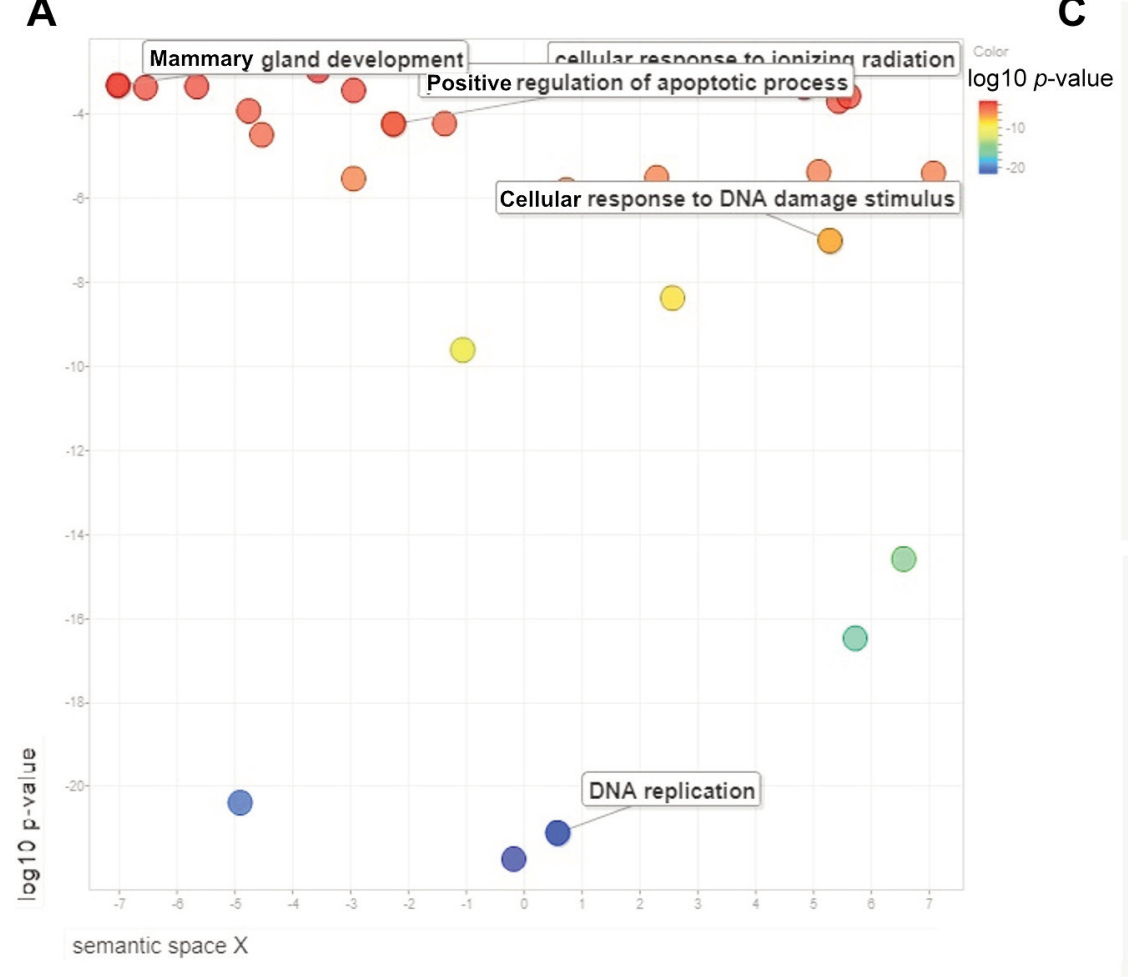

\section{B}

\begin{tabular}{crc}
\hline Index & Name & $\boldsymbol{p}$-Value \\
\hline 1 & ATR signaling pathway & $5.945 \mathrm{e}-15$ \\
2 & DNA-IR damage and celular response via ATR & $2.467 \mathrm{e}-10$ \\
3 & G2/M checkpoints & $6.216 \mathrm{e}-9$ \\
4 & Fanconi Anemia pathway & $1.083 \mathrm{e}-8$ \\
5 & ATR activation in response to replication & $1.527 \mathrm{e}-7$ \\
6 & stress & $2.649 \mathrm{e}-7$ \\
7 & G1/S specific transciption & $2.801 \mathrm{e}-7$ \\
\hline
\end{tabular}

C

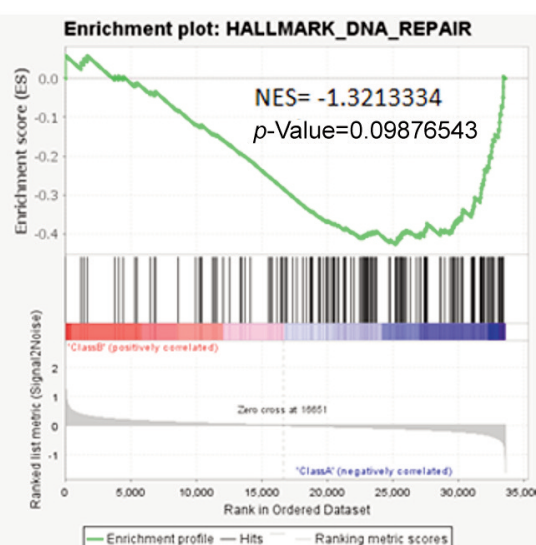

-Enichiment proflio - Hiss - Ranking metric scooes

Enrichment plot: KEGG_CELL_CYCLE

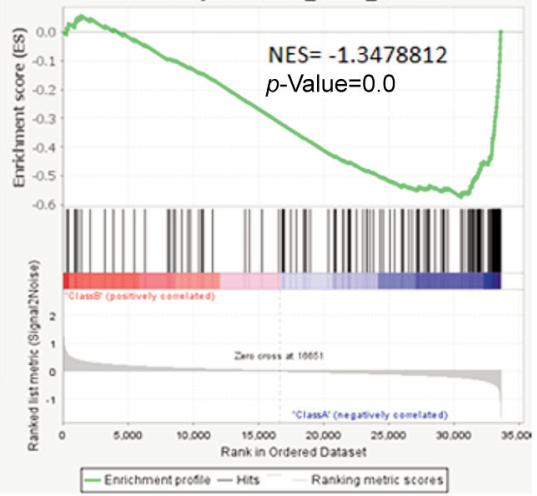

Enrichment plot: HALLMARK_G2M_CHECKPOINT

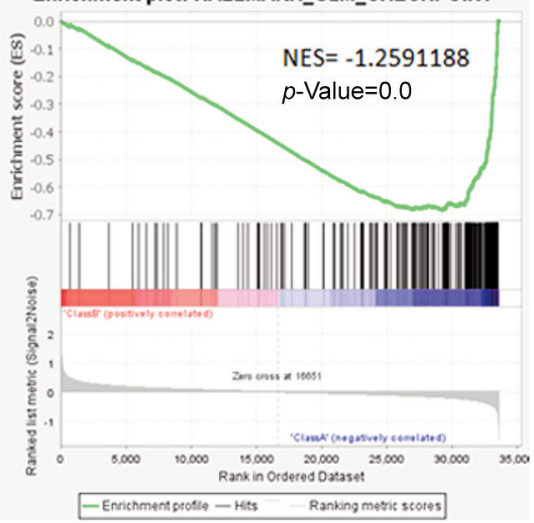

Figure 4. Bioinformatic analysis of long intergenic non-coding RP11-0400k9.4 (lincRP11-400k9.4) overexpression in MCF-7 cells. A and B: Main cellular processes altered by lincRP11-900k.9.4 overexpression analyzed by DAVIDREVIGO software and Gene set enrichment analysis. C: Pathways most altered as analyzed by Key Pathway Advisor (KPA), Metacore and Enrichr software. NES: Normalized enrichment score; ATR: ataxia telangiectasia and RAD3-related; IR: ionizing radiation; G1: gap 1; S: synthesis.

RT-PCR (Figure 3). As shown in Figure 3B, 710 transcripts were deregulated: Levels of 398 increased and 312 decreased, using a fold change of 2 or more and $p<0.05$ as threshold. Table I shows the top 10 altered RNAs. In order to delve into the expression changes mediated by lincRNA-RP11400k9.4, we carried out a network analysis using the KPA, Metacore,
Enrichr, GSEA and REVIGO tools. We found that the main altered processes were related to DNA repair mechanisms, including DNA damage by UV radiation (Figure 4).

To corroborate the role of lincRNA-RP11400k9.4 in DNA repair mechanisms, and in particular DNA damage induced by UV radiation, we exposed MCF-7 and MDA-MB 468 

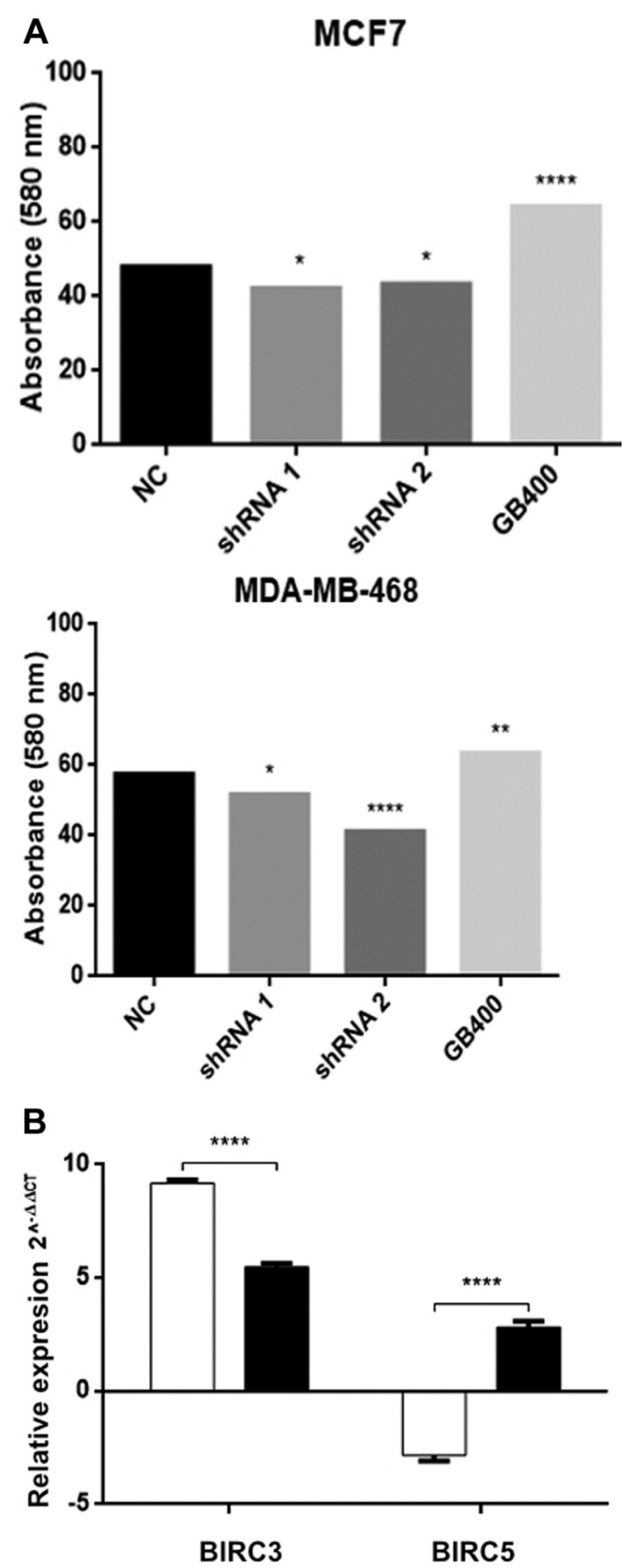

Figure 5. Effect of long intergenic non-coding RP11-0400k9.4 (lincRP11-400k9.4) expression on cellular survival and proliferation. A: Cellular survival after exposure to ultraviolet (UV) radiation of MCF7 (left) and MDA-MB-468 (right) cells overexpressing (GB400) or underexpressing lincRP11-400k9.4. B: Expression of Baculoviral IAP Repeat Containing 3 (BIRC3) and Baculoviral IAP Repeat Containing 3 (BIRC5) in MCF-7 cells overexpressing lincRNA RP11-400k9.4. Short hairpin RNA (shRNA); geneblock for RP11-400k9.4 (GB400). Two-way ANOVA was performed to evaluate the significance of differences. Significantly different at: ${ }^{*} p=0.05, * * p=0.01$, and ${ }^{* * *} p=0.0001$. cells with dysregulated lincRNA-RP11400k9.4 levels to UV light $(254 \mathrm{~nm}$ ) for $3 \mathrm{~min}$. Twenty-four hours later, cellular viability was measured. As shown in Figure 5A, cells with lincRNA-RP11400k9.4 knockdown were more sensitive to UV radiation exposure and, as expected, cells with lincRNARP11400k9.4 overexpression presented higher cellular survival after UV radiation. Since UV light induces apoptosis, we analyzed the RNA data for differentially expressed genes associated with this process. Interestingly, we found that BIRC3, BCL2 apoptosis regulator-like protein 11 (BIM) and survivin (BIRC5) were dysregulated by lincRNA-RP11400k9.4 overexpression. We then measured $B I R C 3$ and BIRC5 expression using RT-PCR and found that the expression of both genes was increased (Figure $5 \mathrm{~B}$ ). It is important to note that in the microarray data only $B I R C 3$ expression increased whereas that of BIRC5 decreased, perhaps due to the presence of multiple functional isoform of this proteins with different regulatory patterns.

Since it has been reported that BIRC3 increases resistance to cell death but at the same time inhibits migration and proliferation, we evaluated the migratory capacity of these cells by transwell assay. As shown in Figure 6, both MCF-7 and MDA-MB-468 cells with knockdown of lincRNARP11400k9.4 expression presented a migration increase. As expected, overexpression of lincRNA-RP11400k9.4 produced a reduction in the migration of $\mathrm{MCF}-7$ cells (Figure 6A-D). Finally, we measured the proliferative capacity using transfected MCF-7 and MDA-MB-468 cells. As shown in Figure 6E, changes in the level of lincRNARP11400k9.4 did not affect the proliferation of these cells.

\section{Discussion}

Cancer consists of at least 100 diseases shaped by an uncontrolled cell growth. Breast cancer in particular, represent the third cause of Mexican cancer-associated deaths for women of 30 years and older and has maintain this status over the past 8 years $(1,26)$.

Twenty-four years ago, with the discovery of the first lncRNA in eukaryotic cells and their important role in cellular physiology, a new paradigm was established and more recently, this paradigm has extended to carcinogenesis. Recently, Mathias et al. found that some lncRNAs are potential markers of breast cancer subtypes (18). More interesting, $\mathrm{Su}$ et al. performed bioinformatical analyses on 658 ductal carcinomas and found several lncRNAs to be differentially expressed between breast cancer subtypes, lincRNA-RP11400k9.4 being one of the RNAs associated with human epidermal growth factor receptor 2 (HER2)overexpressing subtype (19). HER2-overexpressing tumors are characterized by their aggressiveness and short survival time for their carrier. This observation was similar to that of Van Grembergen et al. in a 995 patient cohort where 
A
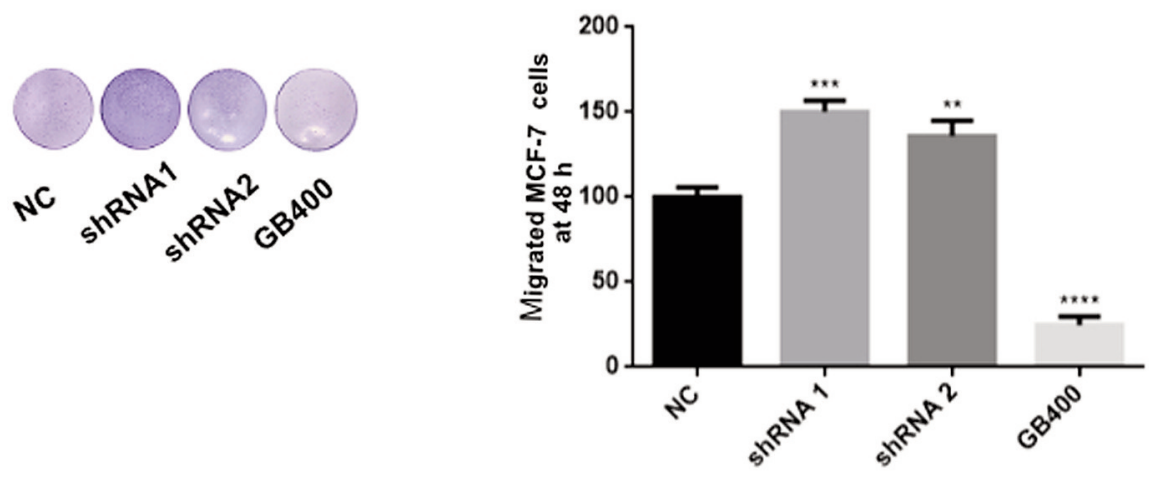

B
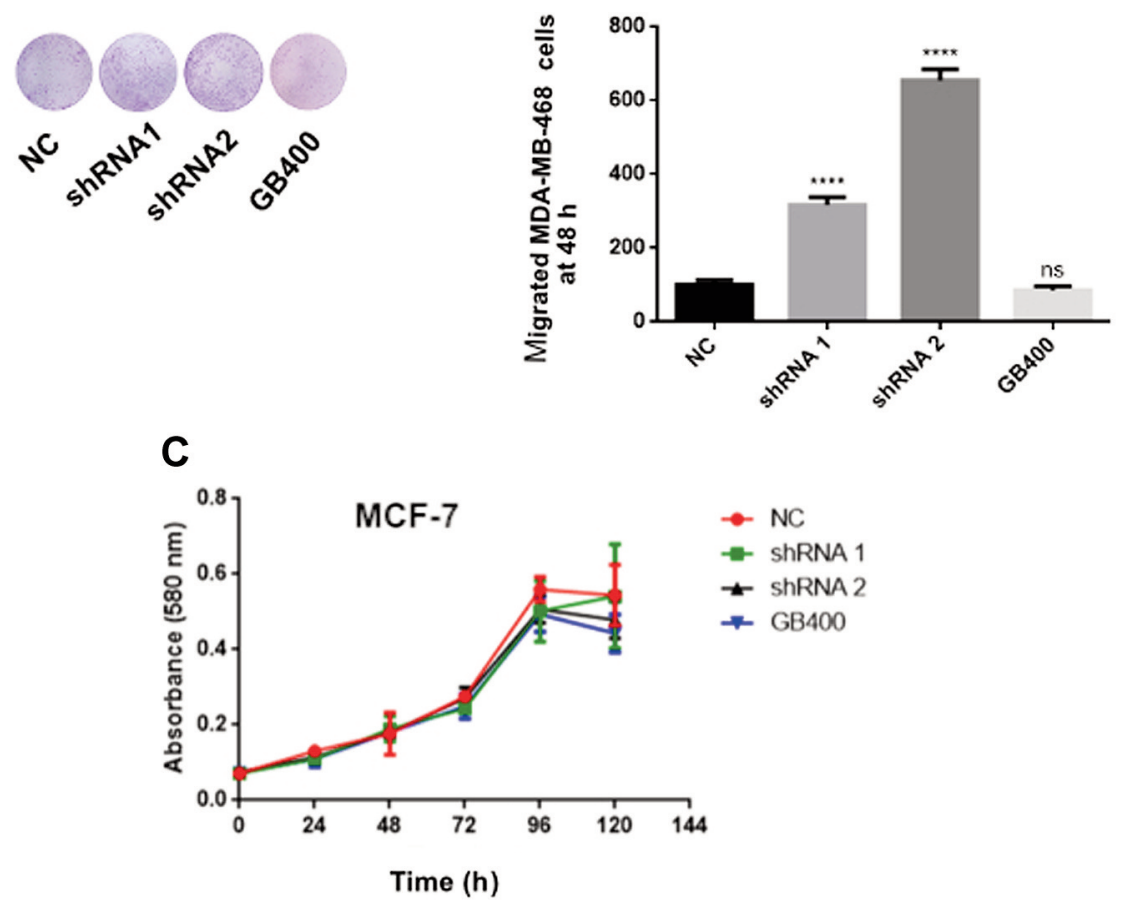

Figure 6. Functional analysis of long intergenic non-coding RP11-0400k9.4 (lincRP11-400k9.4) in breast cancer cells. A: Representative images and quantification of Transwell assay of migration in MCF-7 cells. B: Representative images and quantification of Transwell assay of migration in MDA-MB-468 cells. overexpression of lincRP11-400k9.4 reduced migration of both cell lines. C: MCF-7 proliferation presented no alteration after forced or silenced expression of lincRP11-400k9.4. Short hairpin RNA (shRNA); GeneBlock for RP11-400k9.4 (GB400). All data are shown as the mean \pm SEM from three independent experiments. Student's t-test was performed to evaluate differences. Significantly different from the negative countrol (NC) at: ** $p=0.01, * * * p=0.001, * * * * p=0.0001$.

lincRNA-RP11400k9.4 was also overexpressed in the HER2 subtype (20). We did not find a clear correlation between the level of lincRNA-RP11400k9.4 and HER2-positive status in a panel of breast cancer cell lines. A higher level of this lincRNA was found in the MDA-MB-453 cell line, which is HER2-positive, whereas a lower level was found in MDAMB-361 cells, which are also HER2-positive. In addition, the T47D cell line, which is negative for HER2 showed high levels of lincRNA-RP11400k9.4. These results indicate that alternative pathways may be involved in regulating lincRNARP11400k9.4 expression.

LincRNA-RP11400k9.4 was found to be mainly located in the cytoplastic compartment and was dysregulated in 3D breast cancer cultures and in breast cancer cells that overexpress IKKe. When lincRNA-RP11400k9.4 was overexpressed in breast cancer cells, a relatively large number of RNAs were 
deregulated. Network analyses with data from dysregulated RNAs suggested that lincRNA-RP11400k9.4 may be associated with the ability of cells to survive when exposed to UV radiation. These results were validated with in vitro loss- and gain-of-function approach assays. As expected, overexpression of lincRNA-RP11400k9.4 resulted in a decrease in cell death caused by UV exposure and a decrease in migration but no changes in cell proliferation. BIRC3, a protein that is a member of the NFkappa-B survival pathway, was deregulated in cells that overexpressed lincRNA-RP11400k9.4.

BIRC3 participates in non-canonical nuclear factor kappa $\mathrm{B}(\mathrm{NFkB})$ pathway activation. The activation of NFkB is a commonly observed phenomenon in breast cancer, facilitating the development of a hormone-independent, invasive, high-grade and late-stage tumor phenotype (27). Activated NFkB is associated with resistance to chemotherapy, radiotherapy and endocrine therapy (28).

BIRCs are able to abrogate death-inducing signals, by direct inhibition of caspases and modulation on NFKB (16). Aberrant BIRC3 expression has been found in a several human cancer types. Dierlamm et al. found that the fusion BIRC3-mucosaassociated lymphoid tissue lymphoma protein in lymphoma conferred resistance to apoptosis (29). Yuan et al. reported that the BIRC3 enhanced resistance to apoptosis in HPV16E6/E7immortalized human oral keratinocytes (30). There are reports that point to the role of BIRC3 in the regulation of UV-induced apoptosis: Ismail and Bateman reported that BIRC3 is regulated by T-box transcription factor 2 in SW13 cells and increased resistance to apoptotic stimuli including UV-radiation (31) and it has been reported that BIRC3 was overexpressed in UV- irradiated hepatocytes (32). BIRC3 might mediate the effect of lincRNA-RP11400k9.4,lincRN, perhaps operating downstream of $\mathrm{NFKB}$ and be partly responsible for its effects. Further experiments are needed to address this.

\section{Conclusion}

LincRNA-RP11400k9.4 is an intergenic non-coding RNA, expressed in the cytoplasm of breast cancer cells, which regulates the sensitivity to death after exposure to UV radiation. This lincRNA also inhibits cell migration, although not proliferation in these cells. Its effects may be mediated by BIRC3, a member of the family of IAPs that is regulated by $\mathrm{NFkB}$.

\section{Conflicts of Interest}

The Authors declare that there are no conflicts of interest in regard to this study.

\section{Authors' Contributions}

M.F., J.M., and V.M. contributed to the design and implementation of the research, to the analysis of the results and to the writing of the article.

\section{Acknowledgements}

This work was part of the PhD thesis of Miguel Ángel Fernández Rojas, doctoral student from the Programa de Doctorado en Ciencias Biomédicas, Universidad Nacional Autónoma de México (UNAM) and has received CONACyT fellowship 288752. This study was supported by grants Grant A1-S-8462 to J.M-Z. We greatly acknowledge the technical support of Dra. Karla Vázquez Santillán, Dra. Rosario Castro Oropeza, Dra. Marcela De la Fuente Hernández and Dr. Alfredo García Venzor for the ongoing support.

\section{References}

1 Bray F, Ferlay J, Soerjomataram I, Siegel RL, Torre LA and Jemal A: Global cancer statistics 2018: Globocan estimates of incidence and mortality worldwide for 36 cancers in 185 countries. CA Cancer J Clin 68(6): 394-424, 2018. PMID: 30207593, DOI: $10.3322 /$ caac. 21492

2 Girotti MR, Salatino M, Dalotto-Moreno T and Rabinovich GA: Sweetening the hallmarks of cancer: Galectins as multifunctional mediators of tumor progression. J Exp Med 217(2), 2020. PMID: 7041721. DOI: 10.1084/jem.20182041

3 Hanahan D and Weinberg RA: Hallmarks of cancer: The next generation. Cell 144(5): 646-674, 2011. PMID: 21376230. DOI: 10.1016/j.cell.2011.02.013.

4 Srinivasula SM and Ashwell JD: IAPs: What's in a name? Mol Cell 30(2): 123-135, 2008. PMID: 2677451. DOI: 10.1016/ j.molcel.2008.03.008

5 Birnbaum MJ, Clem RJ and Miller LK: An apoptosis-inhibiting gene from a nuclear polyhedrosis virus encoding a polypeptide with cys/his sequence motifs. J Virol 68(4): 2521-2528, 1994. PMID: 236730. DOI: 10.1128/JVI.68.4.2521-2528.1994

6 Schimmer AD: Inhibitor of apoptosis proteins: Translating basic knowledge into clinical practice. Cancer Res 64(20): 7183-7190, 2004. PMID: 15492230. DOI: 10.1158/0008-5472.CAN-04-1918

7 Miura K, Fujibuchi W, Ishida K, Naitoh T, Ogawa H, Ando T, Yazaki N, Watanabe K, Haneda S, Shibata C and Sasaki I: Inhibitor of apoptosis protein family as diagnostic markers and therapeutic targets of colorectal cancer. Surg Today 41(2): 175182, 2011. PMID: 21264751. DOI: 10.1007/s00595-010-4390-1

8 Mendoza-Rodriguez M, Arevalo Romero H, Fuentes-Panana EM, Ayala-Sumuano JT and Meza I: IL-1beta induces upregulation of $B I R C 3$, a gene involved in chemoresistance to doxorubicin in breast cancer cells. Cancer Lett 390: 39-44, 2017. PMID: 28093282. DOI: 10.1016/j.canlet.2017.01.005

9 Gyrd-Hansen M and Meier P: IAPs: From caspase inhibitors to modulators of NF-kappaB, inflammation and cancer. Nat Rev Cancer 10(8): 561-574, 2010. PMID: 20651737. DOI: $10.1038 / \mathrm{nrc} 2889$

10 Cao J, Qiu J, Wang X, Lu Z, Wang D, Feng H, Li X, Liu Q, Pan $\mathrm{H}$, Han X, Wei J, Liu S and Wang L: Identification of microRNA124 in regulation of hepatocellular carcinoma through BIRC3 and the NF-kappaB pathway. J Cancer 9(17): 3006-3015, 2018. PMID: 6134807. DOI: 10.7150/jca.25956

11 Wang L, Luan T, Zhou S, Lin J, Yang Y, Liu W, Tong X and Jiang W: Lncrna hcp5 promotes triple negative breast cancer progression as a ceRNA to regulate BIRC3 by sponging mir219a-5p. Cancer Med 8(9): 4389-4403, 2019. PMID: 6675706. DOI: $10.1002 / \mathrm{cam} 4.2335$ 
12 Lin K, Song LJ, Ma J, Zhang TS, You DY and He YW: Identification of cancer hallmark-associated gene and lncRNA cooperative regulation pairs and dictate $\operatorname{lncRNA}$ roles in oral squamous cell carcinoma. J Cell Mol Med 24(9): 5213-5223, 2020. PMID: 7205782. DOI: 10.1111/jcmm.15174

13 Deng Y, Luo S, Zhang X, Zou C, Yuan H, Liao G, Xu L, Deng C, Lan Y, Zhao T, Gao X, Xiao Y and Li X: A pan-cancer atlas of cancer hallmark-associated candidate driver lncRNAs. Mol Oncol 12(11): 1980-2005, 2018. PMID: 6210054. DOI: $10.1002 / 1878-0261.12381$

14 Adriaens C, Standaert L, Barra J, Latil M, Verfaillie A, Kalev P, Boeckx B, Wijnhoven PW, Radaelli E, Vermi W, Leucci E, Lapouge G, Beck B, van den Oord J, Nakagawa S, Hirose T, Sablina AA, Lambrechts D, Aerts S, Blanpain C and Marine JC: P53 induces formation of NEAT1 lncRNA-containing paraspeckles that modulate replication stress response and chemosensitivity. Nat Med 22(8): 861-868, 2016. PMID: 27376578. DOI: $10.1038 / \mathrm{nm} .4135$

15 Betts JA, Moradi Marjaneh M, Al-Ejeh F, Lim YC, Shi W, Sivakumaran H, Tropee R, Patch AM, Clark MB, Bartonicek N, Wiegmans AP, Hillman KM, Kaufmann S, Bain AL, Gloss BS, Crawford J, Kazakoff S, Wani S, Wen SW, Day B, Moller A, Cloonan N, Pearson J, Brown MA, Mercer TR, Waddell N, Khanna KK, Dray E, Dinger ME, Edwards SL and French JD: Long noncoding RNAs CUPID1 and CUPID2 mediate breast cancer risk at $11 \mathrm{q} 13$ by modulating the response to DNA damage. Am J Hum Genet 101(2): 255-266, 2017. PMID: 5544418. DOI: 10.1016/j.ajhg.2017.07.007

16 Casimiro S, Alho I, Bettencourt M, Pires R, Lipton A and Costa $\mathrm{L}$ : Rankl enhances the effect of an antagonist of inhibitor of apoptosis proteins (cIAPs) in RANK-positive breast cancer cells. J Bone Oncol 2(3): 116-122, 2013. PMID: 4723389. DOI: 10.1016/j.jbo.2013.07.001

$17 \mathrm{Wu} \mathrm{Z}$ and Wang Y: Studies of lncRNAs in DNA double-strand break repair: What is new? Oncotarget 8(60): 102690-102704, 2017. PMID: 5731991. DOI: 10.18632 /oncotarget.22090

18 Mathias C, Zambalde EP, Rask P, Gradia DF and de Oliveira JC: Long non-coding RNAs differential expression in breast cancer subtypes: What do we know? Clin Genet 95(5): 558-568, 2019. PMID: 30614523. DOI: 10.1111/cge.13502

19 Su X, Malouf GG, Chen Y, Zhang J, Yao H, Valero V, Weinstein JN, Spano JP, Meric-Bernstam F, Khayat D and Esteva FJ: Comprehensive analysis of long non-coding RNAs in human breast cancer clinical subtypes. Oncotarget 5(20): 9864-9876, 2014. PMID: 4259443. DOI: 10.18632/oncotarget.2454

20 Van Grembergen O, Bizet M, de Bony EJ, Calonne E, Putmans P, Brohee S, Olsen C, Guo M, Bontempi G, Sotiriou C, Defrance $\mathrm{M}$ and Fuks F: Portraying breast cancers with long noncoding RNAs. Sci Adv 2(9): e1600220, 2016. PMID: 5010371. DOI: 10.1126/sciadv. 1600220

21 Orlova Z, Pruefer F, Castro-Oropeza R, Ordaz-Ramos A, Zampedri C, Maldonado V, Vazquez-Santillan K and MelendezZajgla J: IKKepsilon regulates the breast cancer stem cell phenotype. Biochim Biophys Acta Mol Cell Res 1866(4): 598611, 2019. PMID: 30615901. DOI: 10.1016/j.bbamcr.2019.01.002

22 Livak KJ and Schmittgen TD: Analysis of relative gene expression data using real-time quantitative PCR and the 2(-delta delta c(t)) method. Methods 25(4): 402-408, 2001. PMID: 11846609. DOI: $10.1006 /$ meth 2001.1262
23 Subramanian A, Tamayo P, Mootha VK, Mukherjee S, Ebert BL, Gillette MA, Paulovich A, Pomeroy SL, Golub TR, Lander ES and Mesirov JP: Gene set enrichment analysis: A knowledgebased approach for interpreting genome-wide expression profiles. Proc Natl Acad Sci USA 102(43): 15545-15550, 2005. PMID: 1239896. DOI: 10.1073/pnas.0506580102

24 Supek F, Bosnjak M, Skunca N and Smuc T: REVIGO summarizes and visualizes long lists of Gene Ontology terms. PLoS One 6(7): e21800, 2011. PMID: 3138752. DOI: 10.1371/journal.pone.0021800

25 Kuleshov MV, Jones MR, Rouillard AD, Fernandez NF, Duan Q, Wang Z, Koplev S, Jenkins SL, Jagodnik KM, Lachmann A, McDermott MG, Monteiro CD, Gundersen GW and Ma'ayan A: Enrichr: A comprehensive gene set enrichment analysis web server 2016 update. Nucleic Acids Res 44(W1): W90-97, 2016. PMID: 4987924. DOI: 10.1093/nar/gkw377

26 Geografía. INdEy: Instituto nacional de estadística y geografía. Estadísticas a propósito del día mundial contra el cáncer (4 de febrero). Datos nacionales. 2016. Available at: https://www. inegi.org.mx/contenidos/saladeprensa/aproposito/2018/cancer2018_ Nal.pdf [Last accessed on 31st August 2020]

27 Wang W, Nag SA and Zhang R: Targeting the NFkappaB signaling pathways for breast cancer prevention and therapy. Curr Med Chem 22(2): 264-289, 2015. PMID: 6690202. DOI: $10.2174 / 0929867321666141106124315$

28 Zhou Y, Eppenberger-Castori S, Eppenberger U and Benz CC: The NFkappaB pathway and endocrine-resistant breast cancer. Endocr Relat Cancer 12(Suppl 1): S37-46, 2005. PMID: 16113098. DOI: $10.1677 /$ erc.1.00977

29 Dierlamm J, Baens M, Wlodarska I, Stefanova-Ouzounova M, Hernandez JM, Hossfeld DK, De Wolf-Peeters C, Hagemeijer A, Van den Berghe $\mathrm{H}$ and Marynen P: The apoptosis inhibitor gene $A P I 2$ and a novel 18q gene, $M L T$, are recurrently rearranged in the $\mathrm{t}(11 ; 18)(\mathrm{q} 21 ; \mathrm{q} 21)$ associated with mucosa-associated lymphoid tissue lymphomas. Blood 93(11): 3601-3609, 1999. PMID: 10339464. DOI: 10.1182/blood.V93.11.3601

30 Yuan H, Fu F, Zhuo J, Wang W, Nishitani J, An DS, Chen IS and Liu X: Human papillomavirus type 16 e 6 and e 7 oncoproteins upregulate c-IAP2 gene expression and confer resistance to apoptosis. Oncogene 24(32): 5069-5078, 2005. PMID: 15856013. DOI: 10.1038/sj.onc.1208691

31 Ismail A and Bateman A: Expression of TBX2 promotes anchorage-independent growth and survival in the p53-negative SW13 adrenocortical carcinoma. Cancer Lett 278(2): 230-240, 2009. PMID: 19216023. DOI: 10.1016/j.canlet.2009.01.006

32 Chopra M, Dharmarajan AM, Meiss G and Schrenk D: Inhibition of UV-C light-induced apoptosis in liver cells by 2,3,7,8-tetrachlorodibenzo- $p$-dioxin. Toxicol Sci 111(1): 49-63, 2009. PMID: 19520675. DOI: 10.1093/toxsci/kfp128

Received July 28, 2020

Revised August 29, 2020 Accepted September 2, 2020 